\title{
TIDAL SEA WATER MEASUREMENT MONITORING SYSTEM
}

\author{
Umar Katu, Achmad Zubair, Sahbuddin A.K \\ Department, Telecommunication Engineering Politeknik Negeri Ujung Pandang \\ Perintis Kemerdekan Km 10, Indonesia
}

\begin{abstract}
The change in receding sea water is to rise or fall in the position of water or sea surface caused by moon influence and sunlight of gravity. There are three sources of interinteraction styles: sea, Sun, and Moon. Ocean waves cause a change in water depth and generate a current vortex that is known as tidal currents, so that wave events estimate is indispensable in coastal navigation. The coastal area sinks when the tide rises and is exposed at low tide. Data retrieval of regular tidal water using manual method using normal measuring device. The use of electronic components is one of the solutions for obtaining modern data. The use of ultrasonic sensors and Arduino as well as mobile phones become modern media to measure sea water level as well as remote monitoring. The system is also equipped with data memory, so when the system can not send SMS data then the data is still stored in memory card on the system. The Data obtained is also accurate as well as time efficiency is very assured that there is no need to manually measure every hour even after every minute as all manual work is done by a mini computer and an ultrasonic sensor. The device design of the tidal water monitoring system in the form of PASUT tools has been successfully designed and this tool can work well in capturing and transmitting the altitude data of tidal seas through the GSM MODEM and displayed on the phone with Excellent tool accuracy so that this tool is said to be operating properly.
\end{abstract}

Keywords: Monitoring system, Arduino, tidal sea water, ultrasonic sensors, GSM MODEM.

Cite this Article: Umar Katu, Achmad Zubair, Sahbuddin A.K, Tidal Sea Water Measurement Monitoring System. International Journal of Civil Engineering and Technology, 11(2), 2020, 79-87.

https://iaeme.com/Home/issue/IJCIET?Volume=11\&Issue=2

\section{INTRODUCTION}

The water at the end of the beach bordering the sea never dwells on a fixed height, but they always move up and down according to the Tide cycle. The surface of the sea water slowly rises to the maximum height, the event is called high Water, after which it drops to a minimum height called low water. From here the water surface will begin to move up again. The difference in height between high tide and low tide is known as tidal range. The 
distinctive nature of the ups and downs of water surfaces occurs twice daily, so there are two high tide periods and two low tide periods.

The tide is mainly due to the attractive style of attraction between the two forces occurring in the ocean derived from centrifugal force caused by the rotation of the Earth in its axis and the gravitational force coming from the moon. The result of this power plant will be found there are two water mass bulges in which one part is found on the surface of the earth closest to the moon and the two other bulges are at the farthest part side From the moon. Positions of the moon, Earth, sun produce waves of spring tides and neap tides

The ups and downs are fluctuating from the high level of seawater in the region, many invoices affecting the ups and downs of the region. Tidal data can be taken directly or using satellite image processing. By conducting a tidal survey we can find out the classification, highest value, lowest value and average ups and downs in a location. Tidal data retrieval done at least 15 days or 1 Pyantan. In the planning of ports, coastal development such as for mining and oil port tidal data is crucial for projections and future planning Sea water tidal observation manually requires very accurate cost and data accuracy

From the background above the author intends to examine and designing the monitoring system the tidal measurements of sea water using ultrasonic sensors microcontroller-based with the security of the measurement data and also send measurement data via SMS. System This tidal monitoring is a system capable of To monitor the high sea water movements or the conditions All the time that includes components or elements that connected together to facilitate the flow of information. Data monitoring is real time and stages in a system monitoring is divided into three process is:

- The process of collecting and storing sea water altitude data.

- Process of displaying result data monitoring.

- Process data delivery results monitoring in real time

\section{SYSTEM AND DESIGN}

\subsection{Arduino Uno}

Arduino Uno is a microcontroller board based on 8-bit ATmega328P microcontroller. Along with ATmega328P, it consists other components such as crystal oscillator, serial communication, voltage regulator, etc. to support the microcontroller. Arduino Uno has 14 digital input/output pins (out of which 6 can be used as PWM outputs), 6 analog input pins, a USB connection, A Power barrel jack, an ICSP header and a reset button.

The 14 digital input/output pins can be used as input or output pins by using pinMode(), digitalRead() and digitalWrite() functions in arduino programming. Each pin operate at $5 \mathrm{~V}$ and can provide or receive a maximum of $40 \mathrm{~mA}$ current, and has an internal pull-up resistor of 20-50 KOhms which are disconnected by default. Out of these 14 pins, some pins have specific functions as listed below:

- Serial Pins 0 (Rx) and 1 (Tx): Rx and Tx pins are used to receive and transmit TTL serial data. They are connected with the corresponding ATmega328P USB to TTL serial chip.

- External Interrupt Pins 2 and 3: These pins can be configured to trigger an interrupt on a low value, a rising or falling edge, or a change in value.

- PWM Pins 3, 5, 6, 9 and 11: These pins provide an 8-bit PWM output by using analog Write() function.

- SPI Pins 10 (SS), 11 (MOSI), 12 (MISO) and 13 (SCK): These pins are used for SPI communication. 
- In-built LED Pin 13: This pin is connected with an built-in LED, when pin 13 is HIGH LED is on and when pin 13 is LOW, its off.

Along with 14 Digital pins, there are 6 analog input pins, each of which provide 10 bits of resolution, i.e. 1024 different values. They measure from 0 to 5 volts but this limit can be increased by using AREF pin with analog Reference () function.

- Analog pin 4 (SDA) and pin 5 (SCA) also used for TWI communication using Wire library.

Arduino Uno has a couple of other pins as explained below:

- AREF: Used to provide reference voltage for analog inputs with analog Reference() function.

- Reset Pin: Making this pin LOW, resets the microcontroller.

Arduino can be used to communicate with a computer, another Arduino board or other microcontrollers. The ATmega328P microcontroller provides UART TTL (5V) serial communication which can be done using digital pin $0(\mathrm{Rx})$ and digital pin 1 (Tx). An ATmega16U2 on the board channels this serial communication over USB and appears as a virtual com port to software on the computer. The ATmega16U2 firmware uses the standard USB COM drivers, and no external driver is needed. However, on Windows, a inf file is required. The Arduino software includes a serial monitor which allows simple textual data to be sent to and from the Arduino board. There are two RX and TX LEDs on the arduino board which will flash when data is being transmitted via the USB-to-serial chip and USB connection to the computer (not for serial communication on pins 0 and 1). A SoftwareSerial library allows for serial communication on any of the Uno's digital pins. The ATmega328P also supports I2C (TWI) and SPI communication. The Arduino software includes a Wire library to simplify use of the $\mathrm{I} 2 \mathrm{C}$ bus.

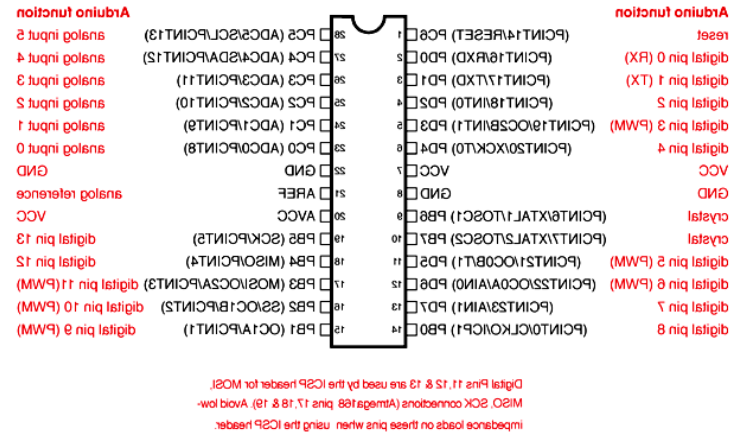

Figure 1 Pin Arduino Uno

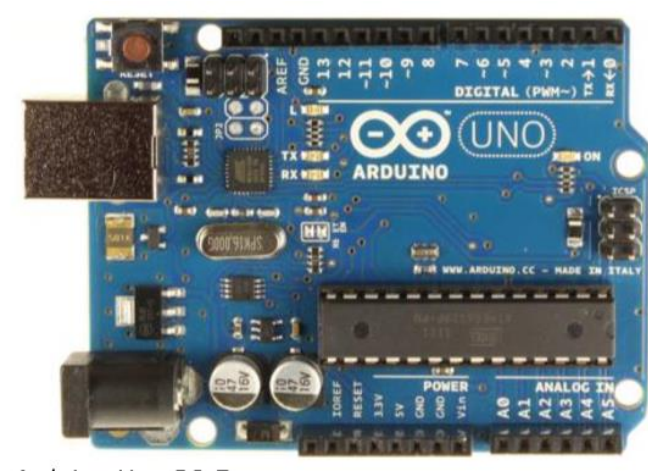

Figure 2 Arduino Uno

\subsection{Ultrasonic Distance Sensor}

The Parallax PING))) ${ }^{\mathrm{TM}}$ ultrasonic distance sensor provides precise, non-contact distance measurements from about $2 \mathrm{~cm}$ ( 0.8 inches) to 3 meters ( 3.3 yards). It is very easy to connect to microcontrollers such as the BASIC Stamp®, Propeller chip, or Arduino, requiring only one I/O pin.

The PING))) sensor works by transmitting an ultrasonic (well above human hearing range) burst and providing an output pulse that corresponds to the time required for the burst echo to return to the sensor. By measuring the echo pulse width, the distance to target can easily be calculated. 


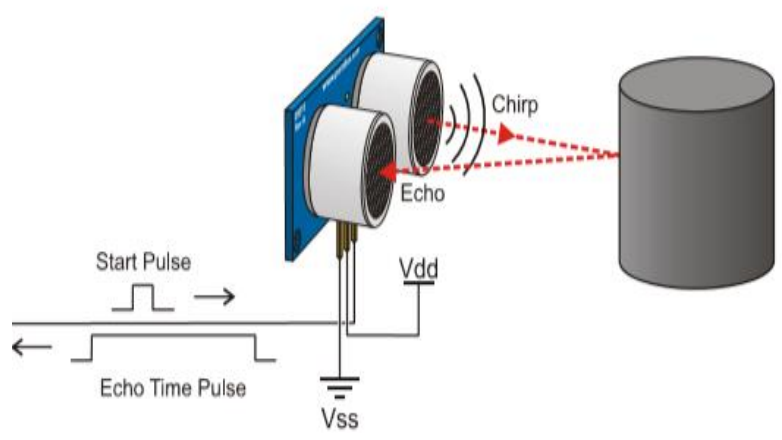

Figure 3 Sensor Ping Parallax

The PING))) sensor has a male 3-pin header used to supply ground, power (+5 VDC) and signal. The header may be plugged into a directly into solderless breadboard, or into a standard 3wire extension cable

\section{Features}

- Range: $2 \mathrm{~cm}$ to $3 \mathrm{~m}(0.8$ in to $3.3 \mathrm{yd})$

- Burst indicator LED shows sensor activity

- Bidirectional TTL pulse interface on a single I/O pin can communicate with $5 \mathrm{~V}$ TTL or $3.3 \mathrm{~V}$ CMOS microcontrollers

- Input trigger: positive TTL pulse, $2 \mu$ s min, $5 \mu$ s typ.

- Echo pulse: positive TTL pulse, $115 \mu$ s minimum to $18.5 \mathrm{~ms}$ maximum.

- RoHS Compliant

The PING))) sensor detects objects by emitting a short ultrasonic burst and then "listening" for the echo. Under control of a host microcontroller (trigger pulse), the sensor emits a short $40 \mathrm{kHz}$ (ultrasonic) burst. This burst travels through the air, hits an object and then bounces back to the sensor. The PING))) sensor provides an output pulse to the host that will terminate when the echo is detected, hence the width of this pulse corresponds to the distance to the target

\subsection{Real Time CLock}

The DS3231 is a low-cost, extremely accurate I2C real-time clock (RTC) with an integrated temperature compensated crystal oscillator (TCXO) and crystal. The device incorporates a battery input, and maintains accurate timekeeping when main power to the device is interrupted. The integration of the crystal resonator enhances the long-term accuracy of the device as well as reduces the piece-part count in a manufacturing line. The DS3231 is available in commercial and industrial temperature ranges, and is offered in a 16-pin, 300-mil SO package. The RTC maintains seconds, minutes, hours, day, date, month, and year information. The date at the end of the month is automatically adjusted for months with fewer than 31 days, including corrections for leap year. The clock operates in either the 24-hour or 12-hour format with an AM/PM indicator. Two programmable time-of day alarms and a programmable square-wave output are provided. Address and data are transferred serially through an $\mathrm{I} 2 \mathrm{C}$ bidirectional bus. A precision temperature-compensated voltage reference and comparator circuit monitors the status of VCC to detect power failures, to provide a reset output, and to automatically switch to the backup supply when necessary. Additionally, the RST pin is monitored as a pushbutton input for generating a $\mu \mathrm{P}$ reset. 


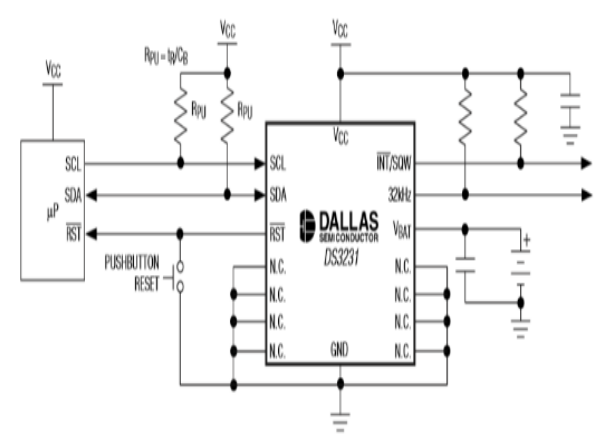

Figure 4 Schematic Ds3230

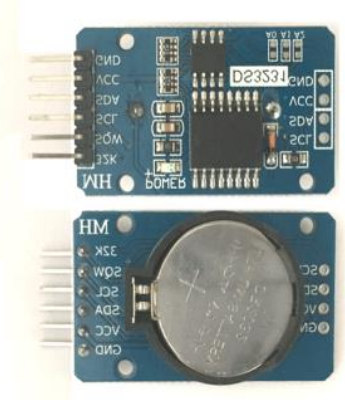

Figure 5 Module Ds3230

This RTC (Real Time Clock) module has very high accuracy and precision in counting time by using RTC IC DS3231 extremely accurate temperature compensated RTC (TCXO). DS3231 has an internal crystal and tuning capacitor circuit in which the temperature of the crystal is monitored continuously and the capacitor is automatically adjusted to maintain a stable pulse rate. Time counting on other RTC solutions can shift (drift) to a matter of minutes per month, especially in conditions of extreme temperature changes. This module most shifts only less than 1 minute per year, thus this module is suitable for critical applications that are sensitive to the accuracy of time which does not need to be synchronized regularly to external clocks. Accessing this module is done through the I2C interface which can be said to be identical to the addressing register on the RTC DS1307, so the program code that has been made for Arduino or other micro-controllers can run without needing to be modified. This module is also equipped with IC AT24C32 which provides an additional EEPROM of $4 \mathrm{~KB}$ (32,768 bits) that can be used for various purposes, for example to save a schedule (time schedule), save alarm time settings, save holiday data on the calendar, record attendance, etc. The address of this EEPROM can be set by shortening pad A0, A1, and A2 (8 address options), by default it is set to address 0x57

\subsection{GSM Shield Simcomm (SIM 900A)}

Sim900A is an additional component as a short message sender used in this study. The Sim900A GSM / GPRS module is the part that functions to communicate between the main monitor and the cell phone. and PT.Satelindo are the two operators of GSM technology pioneers in Indonesia. GSM uses digital technology, there are several advantages of using digital technology compared to analog such as large capacity, better security systems and more diverse services. GSM uses the combined access technology between FDMA (Frequency Division Multiple Access) and TDMA (Time Division Multiple Access) which initially worked at $900 \mathrm{Mhz}$ frequency and this is a standard pioneered by ETSI (The European Telecommunication Standard Institute) where the frequency used with $25 \mathrm{Khz}$ frequency band width at $900 \mathrm{Mhz}$ frequency band. The $25 \mathrm{Khz}$ frequency band is then divided into 124 frequency carriers consisting of $200 \mathrm{Khz}$ each carrier. The $200 \mathrm{Khz}$ frequency carrier is then divided into 8 time slots where each user will make and receive calls in one time slot based on time settings. GSM technology is currently the most widely used in the world and also in Indonesia because one of the advantages of GSM is its extensive roaming capability so it can be used in various countries. However, data access speeds on GSM networks are very small at around $9.6 \mathrm{kbps}$, because initially it was only designed for sound use 


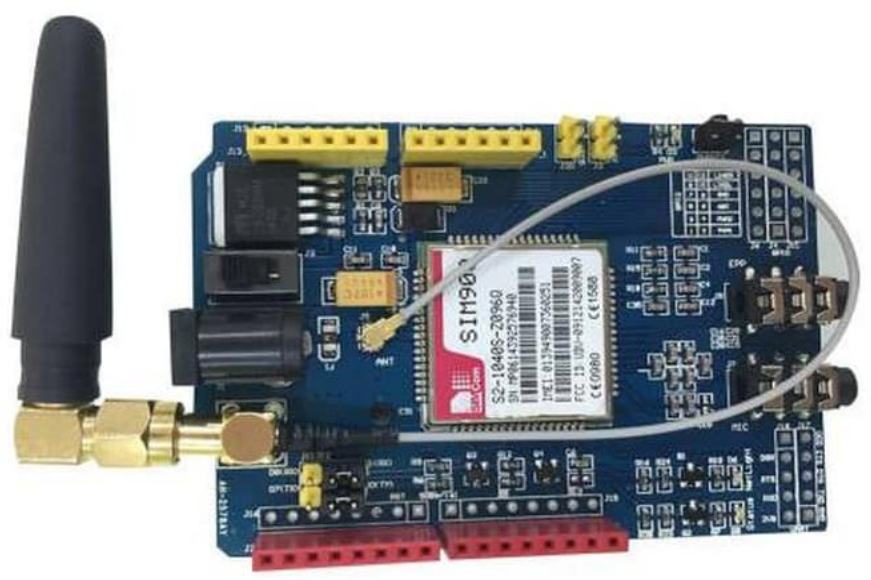

Figure 6 SIM900A Module

\subsection{Liquid Crystal Display (16 x 2)}

LCD (Liquid Crystal Display) is a type of display media that uses liquid crystal as the main viewer. As for the features presented in this LCD are: - Consists of 16 characters and 2 lines. Has 192 saved characters. - There is a programmed character generator. - Can be addressed in 4-bit and 8-bit modes. - Equipped with back light. The initialization process for the arduino pin connected to the LCD pins RS, Enable, D4, D5, D6, and D7, is carried out in the Liquid Crystal row $(2,3,4,5,6,7)$

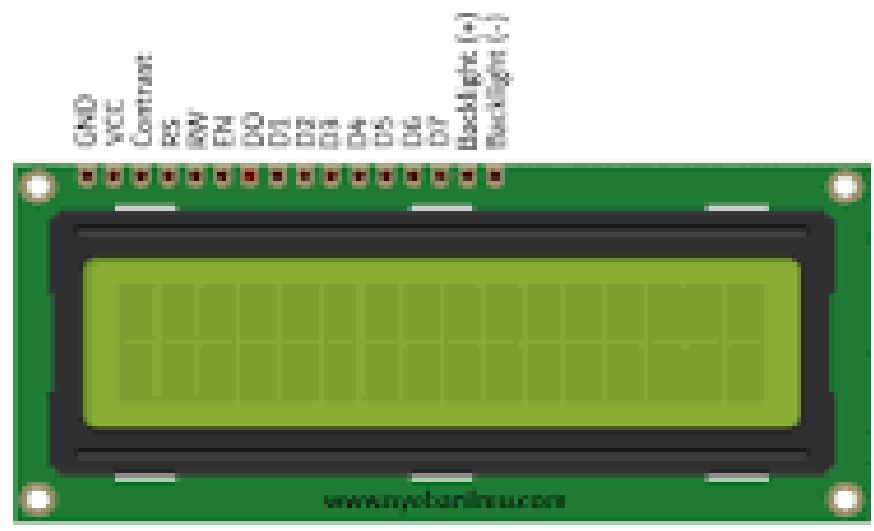

Figure 7 Liquid Crystal Display 16x2

\subsection{System and Design}

The first stage carried out in this research is system design. The system design consists of two parts namely software design (Arduino IDE) and hardware design (System Making).The design of the system to be made in this study is to design a tidal monitoring system using a microcontroller with Arduino Uno as the control center and an Ultrasonic sensor besides the gsm module as the sender of information via sms. The system also contains an LCD device to display measured data to display data parameters as a reference for Tides. The basic concept of this system is by transferring data obtained from the ultrasonic sensor and then sent to the microcontroller and then received to the intended mobile system. The following block diagram diagram shows the system that will be used in the tide monitoring system 


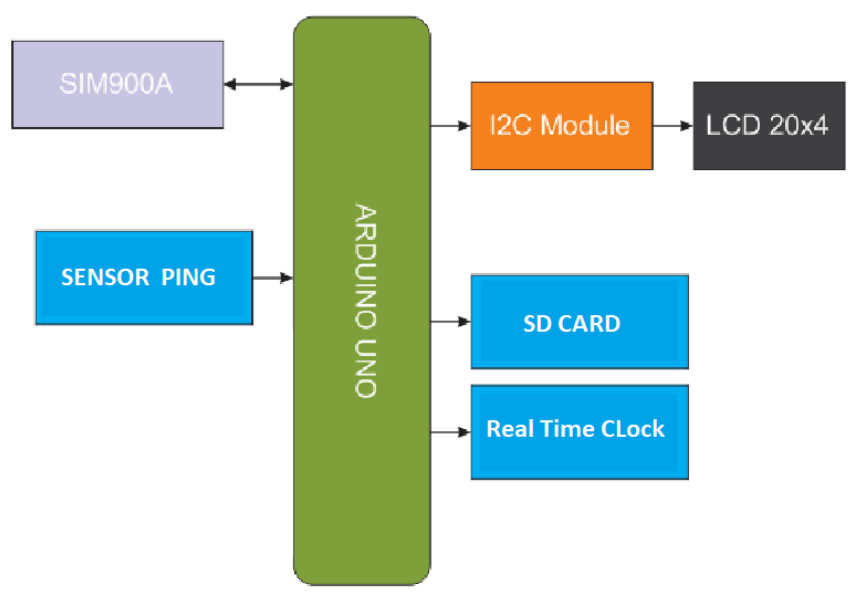

Figure 8 Block Diagram System

\section{SYSTEM OPERATION}

This system works firstly takes ultrasonic sensor data then displays the reading results on the display. The system will save the ultrasonic sensor distance reading data on the sd card module in accordance with the time given by the Real Time Clock (RTC) module, SMS sending time will be determined by how long the duration of time programmed by a system, the duration of time for sending Short Message Service (SMS) can be set as needed starting from 5 minutes to 60 minutes for 1 time sending SMS. Wiring diagram system can be seen in the following picture

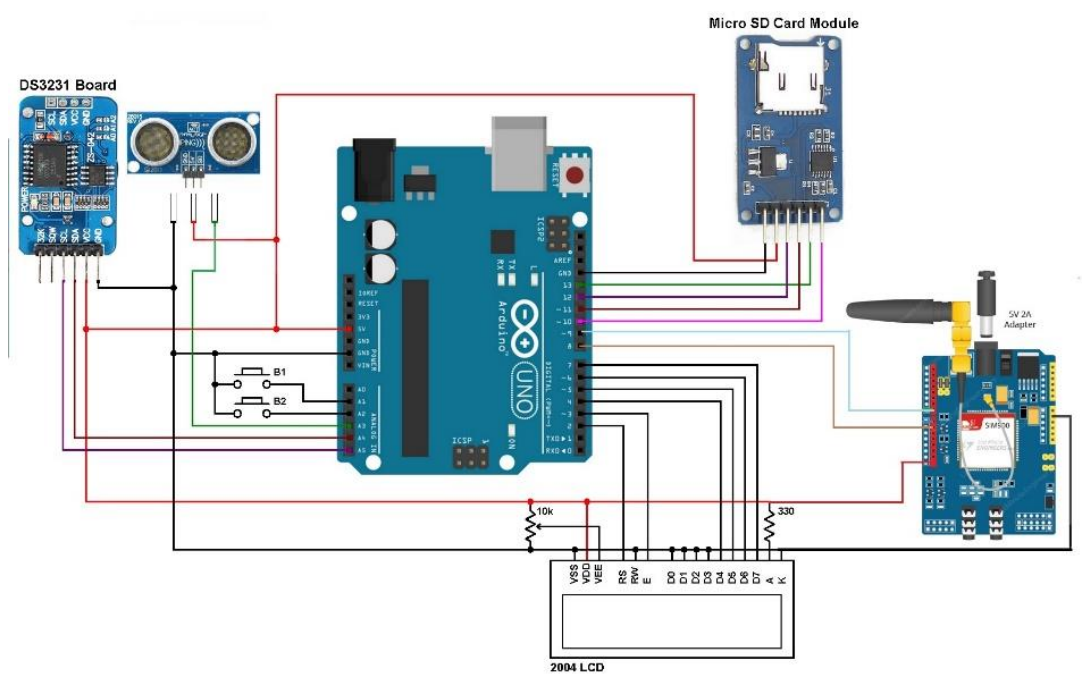

Figure 9 Design Sytem Tidal Monitoring

\section{RESULT AND DISCUSSION}

From this System Design, it is needed some tests including the testing of the Ping Parallax sensor to the reflection using water and the experiment table 
Tidal Sea Water Measurement Monitoring System

Table 1 Parallax Ping sensor measurement

\begin{tabular}{|c|c|c|}
\hline Disared Distance & $\begin{array}{c}\text { average distance } \\
\text { measurement results }\end{array}$ & Error \\
\hline 30 & 30,21 & 0,67 \\
\hline 40 & 40,31 & 0,75 \\
\hline 50 & 50,62 & 1,20 \\
\hline 60 & 60,11 & 1,83 \\
\hline 70 & 70,91 & 1,29 \\
\hline 80 & 81,3 & 1,62 \\
\hline 90 & 90,71 & 0,78 \\
\hline 100 & 100,9 & 0,90 \\
\hline 110 & 111,5 & 1,36 \\
\hline 120 & 122,1 & 2,16 \\
\hline 130 & 132,3 & 2,15 \\
\hline 140 & 142,2 & 1,57 \\
\hline 150 & 152,7 & 1,80 \\
\hline 160 & 163,3 & 2,06 \\
\hline 170 & 174,1 & 2,35 \\
\hline 180 & 183,7 & 2,05 \\
\hline 190 & 194,2 & 2,21 \\
\hline 200 & 204,7 & 2,35 \\
\hline 210 & 212,1 & 2,41 \\
\hline 230 & 232,4 & 2,43 \\
\hline 250 & 253,1 & 2,47 \\
\hline 300 & 304,2 & 3,12 \\
\hline
\end{tabular}

From the sensor measurement data table above, it can be concluded that the percentage of sensor errors is very small, namely around 3\% of the reflected media used in the above experiment is using water, the measurements are repeated repeatedly so as to obtain valid data

The next test is how long is the duration of response time for sending SMS and receiving SMS The results of measuring the duration of sms using the Several provider can be seen in the following table sending SMS is done every 5 minutes to send SMS

Table 2 Time Received SMS

\begin{tabular}{|c|c|c|c|c|}
\hline \multirow{2}{*}{ Trial } & \multicolumn{4}{|c|}{ Provider } \\
\cline { 2 - 5 } & $\mathbf{X L}$ & Telkomsel & $\begin{array}{c}\text { Indosat } \\
\text { (Mentari) }\end{array}$ & Axis \\
\hline 1 & 5,71 & 2,1 & 6,1 & 4,1 \\
\hline 2 & 6,3 & 2,3 & 6,3 & 5,2 \\
\hline 3 & 5,53 & 1,9 & 7,2 & 5,6 \\
\hline
\end{tabular}

From the test in table 1, it can be analyzed that the response time for sending SMS is not stable. This is caused by timeliness SMS Center for sending SMS to mobile user. GSM network signal is also very affect the SMS response time, usually GSM networks are very influential in an area certain. If the area is far from the satellite transmitter GSM, the network signal will also be more difficult got. Successfully tested on other GSM numbers as in numbers Halo, XL, Axis, and Indosat (Mentari)

The following photos are the results of the installation of the system for tidal surveys that can work well 


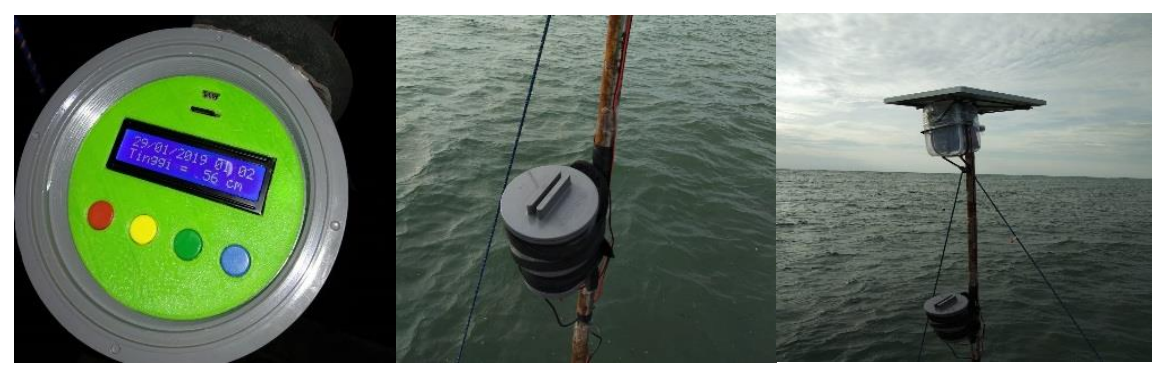

Figure 10 System Tidal Monitoring System

\section{CONCLUDING REMARKS}

From the results of making a tidal monitoring system it can be concluded is :

- The Ultrasonic Sensor can work well with a fairly small error percentage of around $2 \%$

- The duration of time needed to receive a text is fast enough, still under 10 seconds

- SD card memory storage is quite reliable data format can also be made in the form of .txt or even in the form of xls data types.

\section{REFERENCES}

[1] Talukder, "Attacks and defenses in mobile ip: Modeling with stochastic game petri net," in 2017 International Conference on Current Trends in Computer, Electrical, Electronics and Communication (ICCTCEEC), September 2017, pp. 1-8

[2] E. Tate and K. Cuawenberghs, "An innovative ood forecasting system for the Demer basin: A case study," Intl. J. River Basin Management, vol. 3, no. 4, pp. 1- 5, 2005.

[3] E. Kuantama, L. Setyawan, and J. Darma, "Early flood alerts using short message service (SMS)," in International Conference on System Engineering and Technology, Bandung, Indonesia, 2012, pp. 1 - 5.

[4] W. A. H. W. M. Asmara and N. H. A. Aziz, "SMS flood alert system," in IEEE Control and System Graduate Research Colloquium, Shah Alam, Malaysia, Jun. 2011, pp. 18 - 22.

[5] I. Aziz, I. A. Hamizan, N. S. Haron, and M. Mehat, "Cooperative flood detection using GSMD via SMS," in International Symposium on Information Technology (ITSim), Kuala Lumpur, Malaysia, Aug. 2008,pp. 1 - 7.

[6] A. A. Samjani, "General packet radio service [GPRS]," otentials, IEEE, vol. 21, no. 2, pp. $12-15,2002$.

[7] E. Karlsson and K. Persson, "Analysis of hydrometeorological measurements in Tuy Loan river basin, Vietnam," Master of Science Thesis, Lund University, 2005

[8] Ping Ultrasonic Sensor datasheet, : https://www.parallax.com/sites/default/files/downloads/28015-PING-Sensor-ProductGuide-v2.0.pdf

[9] Arduino Uno datasheet, : https://www.fecegypt.com/uploads/dataSheet/

[10] RTC DS3231 datasheet, https://datasheets.maximintegrated.com/en/ds/DS3231.pdf

[11] SIM900A https://components101.com/sites/default/files/component_datasheet/SIM900A 